\title{
A depressão na esquizofrenia
}

Rodrigo A Bressan

Section of Neurochemical Imaging, Institute of Psychiatry, London, UK. Departamento de Psiquiatria da Unifesp/EPM

\section{Introdução}

Embora o diagnóstico da esquizofrenia esteja baseado nos sintomas positivos e negativos, os sintomas depressivos são bastante freqüentes e têm grande importância para os pacientes. As descrições clássicas da esquizofrenia de Kraeplin e Bleuler já enfatizavam a importância dos sintomas depressivos na evolução da doença. ${ }^{1}$ Como os sintomas depressivos não tinham utilidade para a classificação diagnóstica, o estudo desses foi por um longo tempo relegado a um segundo plano. Nas décadas de 60 e 70, com a disseminação de conceitos psicanalíticos na prática psiquiátrica, houve um interesse pelos sintomas depressivos que ocorrem no período que segue a remissão do episódio psicótico agudo. Esses quadros foram denominados de depressão pós-psicótica e eram considerados um sinal de bom prognóstico, bem como uma fase favorável para o trabalho psicoterapêutico. ${ }^{2} \mathrm{O}$ quadro de depressão pós-psicótica tornou-se bastante conhecido e teve grande influência nas classificações diagnósticas vigentes.

Com a introdução dos instrumentos padronizados na psiquiatria, foram encontradas taxas expressivas de depressão em todas as fases da esquizofrenia. Com isso, modificaram-se alguns conceitos associados ao quadro de depressão póspsicótica, como a noção de que os sintomas depressivos são um fenômeno restrito ao período pós-psicótico, associado a bom prognóstico.

\section{Sintomas depressivos no curso da esquizofrenia}

Análises fatoriais conduzidas em grandes amostras têm considerado a depressão uma das dimensões da esquizofrenia, ao lado dos sintomas positivos e negativos. ${ }^{3}$ Os sintomas depressivos ocorrem em todas as fases da doença, especialmente no período prodrômico, $60 \%{ }^{4}$, e durante o episódio psicótico, $75 \%{ }^{5}$ Eles tendem a diminuir de intensidade com o abrandamento dos sintomas positivos, sendo notados no período pós-psicótico $2 \%$ a $15 \%{ }^{5,6}$ Os sintomas depressivos também ocorrem em pacientes estáveis, e as taxas de ocorrência são bem superiores às da população normal. ${ }^{7}$ A freqüência de episódios depressivos em pacientes esquizofrênicos estáveis é bastante alta, variando entre 16,5\% em estudos transversais ${ }^{1}$ e $65 \%$ em estudos de seguimento de 3 anos. ${ }^{8}$ (Figura)

\section{Depressão e prognóstico}

Estudos longitudinais têm considerado a depressão como um indicador de prognóstico desfavorável, associando-a à pior resposta a medicações e ao pior desempenho social, ${ }^{9}$ maiores taxas de recaída e hospitalizações mais longas. ${ }^{8}$ A depressão tem sido associada a uma pior qualidade de vida, ${ }^{10}$ prejuízo de funções cognitivas ${ }^{11}$ e suicídio. Aproximadamente $10 \%$ dos pacientes com esquizofrenia cometem suicídio, e em mais de $60 \%$ desses pacientes o suicídio está associado a sintomas depressivos. ${ }^{12}$

\section{Quadros depressivos na esquizofrenia}

Para se fazer diagnóstico de depressão em pacientes com esquizofrenia é necessário estar atento e investigar ativamente o humor do paciente. Os sintomas depressivos podem ser facilmente confundidos com sintomas negativos ou sintomas extrapiramidais induzidos pelos antipsicóticos. Na esquizofrenia, os sintomas depressivos de maior especificidade e, portanto, de maior utilidade para o diagnóstico clínico são os seguintes: humor deprimido, desesperança, autodepreciação, idéias de referência de culpa, culpa patológica, depressão matutina, despertar precoce e suicídio. ${ }^{13}$ Sintomas como perda de interesse e de prazer, abulia, dificuldade para concentração e alterações do apetite têm pouca utilidade para a avaliação do humor em pacientes com esquizofrenia. Para pesquisa, sugere-se o uso de escalas específicas como a Escala Calgary de depressão para esquizofrenia, traduzida e validada para uso no Brasil..$^{1,14}$

Didaticamente, pode-se dividir os quadros depressivos entre aqueles que ocorrem durante o episódio psicótico e aqueles do período de estabilidade (Tabela). A depressão intrínseca ao episódio psicótico agudo ocorre em aproximadamente $67 \%$ dos pacientes, e tende a regredir juntamente com os sintomas psicóticos positivos. ${ }^{6}$ Em contrapartida, a disforia aguda é um quadro episódico que ocorre no período de estabilidade e se caracteriza por sintomas depressivos e de ansiedade bastante intensos. Freqüentemente está associada a um fator desencadeante, como mudança de ambiente, conflitos familiares etc. O quadro geralmente cede em três semanas, mas pode evoluir para um episódio psicótico agudo ou para um episódio depressivo propriamente dito.

O episódio depressivo da esquizofrenia assemelha-se a um episódio depressivo dos pacientes com transtorno afetivo, com a peculiaridade de que os pacientes têm um quadro psicótico de fundo. O diagnóstico deve se basear na intensidade e duração (mais de duas semanas) dos sintomas específicos de depressão em esquizofrenia, além do impacto ocasionado na vida dos pacientes. O quadro é chamado de depres- 


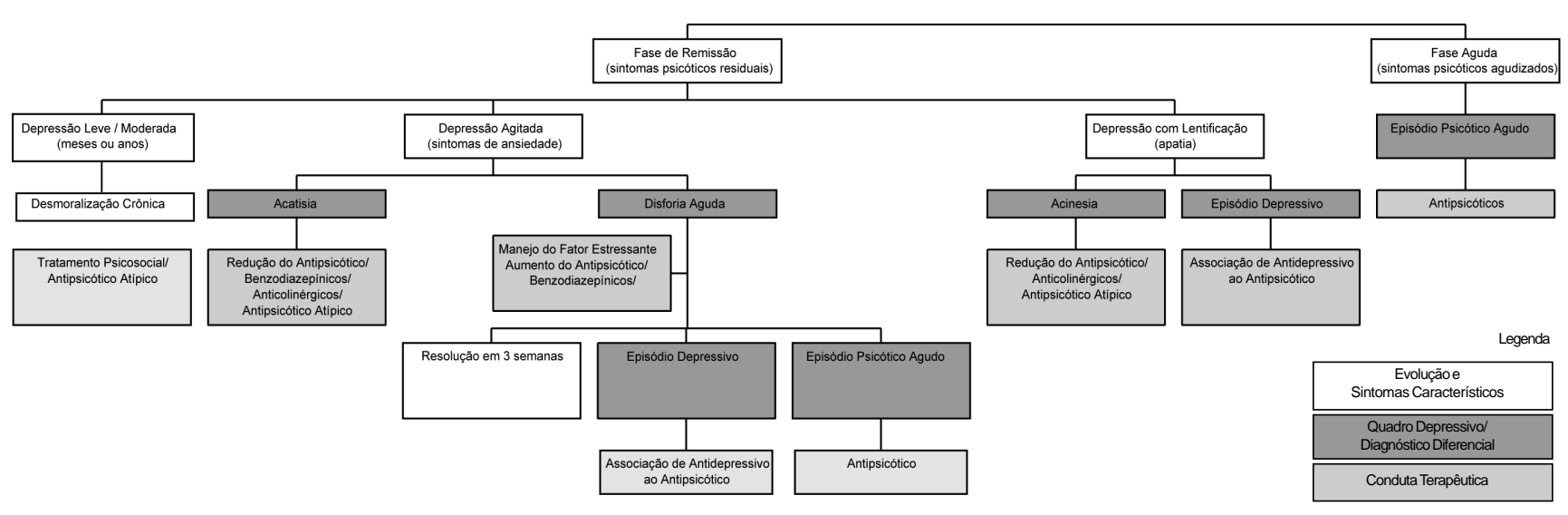

Figura - Sintomas depressivos na esquizofrenia

\section{Tabela - Quadros depressivos na esquizofrenia}

1. Quadro clínico

A. Depressão intrínseca ao episódio psicótico agudo

B. Sintomas depressivos sem sintomas psicóticos agudos Disforia aguda

Episódio depressivo da esquizofrenia

Desmoralização crônica

2. Diagnóstico diferencial

A. Sintomas depressivos secundários a fatores orgânicos

- Efeitos colaterais de antipsicóticos (ex: acatisia e acinesia)

- Transtornos relacionados a substâncias

- Outras causas orgânicas de depressão

B. Outros sintomas da esquizofrenia

- Sintomas negativos (ex: retraimento social, distanciamento afetivo, apatia e anedonia)

- Sintomas inespecíficos (ex: falta de asseio pessoal, insônia, inapetência e lentificação motora)

C. Sintomas depressivos em outros diagnósticos

- Transtorno esquizoafetivo

- Outros transtornos psicóticos (ex: transtorno delirante)

são pós-psicótica da esquizofrenia no DSM-IV e depressão pós-esquizofrênica na CID-10. Como se pôde demonstrar, os episódios depressivos ocorrem tanto na fase pós-psicótica como no período de estabilidade, havendo, portanto, um problema com a nomenclatura atual, que associa a depressão com o período pós-psicótico. ${ }^{15}$

No quadro de desmoralização crônica os sintomas depressivos são mais leves e prolongados, e cursam com bastante desesperança e com risco de suicídio importante. ${ }^{9}$ Esses quadros são entendidos como uma resposta ao estresse real e permanente ocasionado pelo quadro psicótico, embora haja a suspeita de serem conseqüências de alterações fisiopatológicas da esquizofrenia.

\section{Diagnóstico diferencial de depressão em esquizofrenia}

Alguns sintomas negativos, como afastamento social, apatia e anedonia, fazem interseção com sintomas depressivos. O uso de sintomas específicos de depressão é a melhor forma de se evitar essa sobreposição de sintomas (Tabela).

Sintomas extrapiramidais induzidos por antipsicóticos são facilmente confundidos com sintomas depressivos. A acinesia é o quadro que mais se assemelha à depressão e é menos diagnosticado, pois os sintomas extrapiramidais mais típicos, como hipertonia e sialorréia, não estão presentes. ${ }^{16}$ Caracteriza-se por anergia, diminuição da mímica facial e da iniciativa. Siris ${ }^{18}$ recomenda que, antes de se fazer o diagnóstico de depressão, deve-se realizar um teste terapêutico, diminuindo o antipsicótico ou introduzindo um anticolinérgico (até $10 \mathrm{mg}$ de biperideno) por 3 semanas. A acatisia também pode ser facilmente confundida com a disforia aguda, devido à sensação subjetiva de inquietação e agitação motora.

Alguns estudos sugerem que os antipsicóticos típicos podem induzir depressão em pacientes com esquizofrenia, mas essa é uma questão controversa na literatura. ${ }^{19}$ Recentemente, pudemos demonstrar que a intensidade do bloqueio dos receptores $\mathrm{D}_{2}$ de dopamina, provocado pelos antipsicóticos típicos, está relacionada com a ocorrência de sintomas depressivos em pacientes com esquizofrenia. ${ }^{20}$

O diagnóstico diferencial com transtorno esquizoafetivo deve se basear na presença de sintomas afetivos por uma porção substancial da duração total da doença, tanto na fase ativa como na fase residual. Os pacientes que em algumas fases de sua patologia apresentam sintomas depressivos com duração limitada devem permanecer com o diagnóstico de esquizofrenia.

\section{Tratamento}

Para melhor tratar os pacientes psicóticos com sintomas depressivos é preciso identificar com precisão qual o tipo de depressão (Figura). Para tanto, é necessário acompanhamento rigoroso. Como regra geral, pacientes psicóticos devem ser tratados por equipes multidisciplinares em sintonia com os familiares. Todas as questões concernentes ao manejo clínico devem ser discutidas entre a equipe multidisciplinar, os pacientes e suas famílias. De acordo com a gravidade da situação, medidas como intensificação dos atendimentos, 
vigilância constante, controle das medicações, internações em hospital-dia ou em unidades hospitalares devem ser aventadas, mas elas só trarão um real benefício para o paciente se forem tomadas em conjunto. ${ }^{21}$

A depressão intrínseca ao episódio psicótico agudo deve ser tratada da mesma forma que o episódio psicótico, ou seja, com antipsicóticos. Os sintomas depressivos tendem a regredir com a melhora dos sintomas psicóticos. A associação de medicação antidepressiva ao antipsicótico não melhora a resposta terapêutica. ${ }^{17}$ Caso os sintomas depressivos dificultem o manejo do episódio psicótico, sugere-se a introdução de um antipsicótico atípico. Essas medicações estão associadas a uma menor incidência de sintomas depressivos do que os antipsicóticos típicos, seja pela menor afinidade pelos receptores $\mathrm{D}_{2}$ de dopamina ${ }^{20}$ seja pela alta afinidade pelos receptores $5 \mathrm{HT} 2{ }_{\mathrm{A}} \cdot{ }^{21}$ Caso haja um risco importante de suicídio, a internação é uma medida fundamental.

$\mathrm{Na}$ suspeita de um quadro disfórico, deve-se investigar um fator estressor desencadeante e tentar manejá-lo da melhor forma possível. A associação de medicação benzodiazepínica pode ser útil. Em geral, esses quadros regridem em 3 semanas. Entretanto, deve-se estar atento à possível evolução para um episódio psicótico ou para um episódio depressivo da esquizofrenia. Em caso de aumento dos sintomas positivos, é necessário aumentar-se a medicação antipsicótica para prevenir/tratar a recaída.

Os episódios depressivos da esquizofrenia, incluindo a depressão pós-psicótica, devem ser tratados com antidepressivos depois de descartada a acinesia. Os poucos ensaios clínicos controlados avaliando essa questão sugerem que uma parte dos pacientes responde bem a essas medicações. ${ }^{17}$ Devem-se utilizar doses terapêuticas de antidepressivos associadas ao antipsicótico de manutenção por pelo menos 6 semanas. $\mathrm{O}$ antidepressivo mais utilizado nos ensaios clínicos foi a imipramina, mas os inibidores seletivos da recaptação da serotonina podem ser uma boa opção. É importante estar atento às possíveis interações medicamentosas, em particular à disputa por vias metabólicas hepáticas, que podem provocar uma elevação dos níveis séricos das medicações.

Devido à desesperança, os pacientes com desmoralização crônica têm um risco de suicídio importante e, em geral, respondem mal à terapêutica antidepressiva. Não existem ensaios clínicos controlados avaliando essa condição, mas há indicação de que antipsicóticos atípicos podem ser benéficos. ${ }^{19}$ Esses quadros devem ser abordados por meio de medidas psicossociais, cabendo ao psiquiatra estreitar o vínculo com o paciente, procurando aumentar a sua auto-estima. O encaminhamento para psicoterapia ou terapia ocupacional, em grupo ou individual, é medida de grande utilidade. Finalmente, é preciso trabalhar a pertinência desses pacientes na família com orientação e terapia familiar, se necessário.

\section{Conclusão}

Sintomas depressivos são bastante freqüentes em todas as fases da esquizofrenia. Estão associados a vários aspectos negativos do desfecho clinico, incluindo maiores taxa de recaídas, pior qualidade de vida e suicídio. Para se fazer o diagnóstico, deve-se investigar ativamente os sintomas depressivos que sejam específicos de depressão em esquizofrenia. $\mathrm{O}$ acompanhamento dos pacientes deve ser rigoroso, envolvendo equipe multidisciplinar e familiares. A estratégia terapêutica deve se basear no tipo de depressão apresentada pelo paciente. Estudos recentes sugerem que os antipsicóticos atípicos podem ser um bom recurso terapêutico para pacientes com esquizofrenia que apresentam sintomas depressivos. No entanto, ainda não foi comprovada a eficácia desses medicamentos no episódio depressivo da esquizofrenia.

Fonte de financiamento: o autor recebe bolsa de doutorado da Capes.

\section{Referências}

1. Bressan RA, Chaves AC, Shirakawa I, Mari JJ. Validity study of the Brazilian version of the calgary depression scale for schizophrenia. Schizophr Res 1998;32: 41-9.

2. McGlashan TH, Carpenter WT. Postpsychotic depression in schizophrenia. Arch Gen Psychiatry 1976a;33:231-9.

3. White L, Harvey PD, Opler L, Lindenmayer JP, The Panss Study Group. Empirical assessment of the clinical symptoms in schizophrenia. Psychopathol 1997;30:263-74.

4. Herz MI, Melville C. Relapse in schizophrenia. Am J Psychiatry 1980;137(7):801-5.

5. Koreen AR, Siris SG, Chakos M. Depression in first-episode schizophrenia. Am J Psychiatry 1993;150:1643-8.

6. Knights A, Hirsch SR. 'Revealed' depression and drug treatment for schizophrenia. Arch Gen Psychiatry 1981;38:806-11.

7. Zisook S, McAdams LA, Kuck J, Harris MJ, Bailey A, Patterson TL, et al. Depressive symptoms in schizophrenia. Am J Psychiatry 1999;156(11):1736-43.
8. Johnson DAW. The significance of depression in the prediction of relapse in chronic schizophrenia. Br J Psychiatry 1988;152:320-3.

9. Bartels SJ, Drake RE. Depressive symptoms in schizophrenia: comprehensive differential diagnosis. Compr Psychiatry 1988;29:467-83.

10.Tollefeson GD, Andersen SW. Should we consider mood disturbance in schizophrenia as an important determinant of quality of life? J Clin Psychiatry 1999;60Supl 5:23-9.

11.Brebion G, Smith MJ, Amador X, Malaspina D, Gorman JM. Clinical correlates of memory in schizophrenia: differential links between depression, positive and negative symptoms, and two types of memory impairment. Am J Psychiatry 1997;154(11):1538-43.

12.Roy A. Relationship between depression and suicidal behavior in schizophrenia. In: DeLisi LE, editor. Depression in schizophrenia. Washington (DC): American Psychiatric Press; 1990.

13. Addington D, Addington J, Schissel B. A depression rating scale for schizophrenics. Schizophr Res 1990;3:247-51. 
14.Bressan RA, Chaves AC, Shirakawa I, Mari JJ. Reliability study of the portuguese version of the calgary depression rating scale to schizophrenia. Rev ABP-APAL 1997;19(4):149-54.

15. Bressan RA, Chaves AC, Pilowsky LS, Shirakawa I, Mari JJ. Depressive episodes in schizophrenia: a post psychotic phenomena? Schizophr Res 2000;41:47.

16. Van Putten T, May PRA. 'Akinetic depression' in schizophrenia. Arch Gen Psychiatry 1978;35:1101-7.

17.Siris SG. Pharmachological treatment of depression in schizophrenia. In: DeLisi LE, editor. Depression in schizophrenia. Washington (DC): American Psychiatric Press; 1990.

18.Siris SG. Diagnosis of secundary depression in schizophrenia: implications for DSM - IV. Schizophr Bull 1991;17:75-98.
19.Tollefeson GD, Sanger RM, Lu Y, Thieme ME. Depressive signs and symptoms in schizophrenia: a prospective blinded trial of olanzapine and haloperidol. Arch Gen Psychiatry 1998;33:250-8.

20. Bressan RA, Mulligan RS, Costa DC, Jones HM, Ell PJ, Kerwin RW, et al. Striatal D2 blockade and depression in schizophrenia. Biol Psychiatry 2000;47(58):129.

21. Shirakawa I. O ajustamento social na esquizofrenia. São Paulo: Lemos; 1992.

22.Collaborative Working Group On Clinical Trial Evaluations. Atypical antipsychotics for treatment of depression in schizophrenia and affective disorders. J Clin Psychiatry 1998;59Supl 12:41-5. 